Sección II: Notas 



\title{
ALGUNAS NOTAS SOBRE LA PARTICIPACIÓN DE ROGELIO DE TOSNY EN LA RECONQUISTA IBÉRICA
}

\author{
Lucas VILLEGAS ARISTIZÁBAL \\ Universidad de Nottingham
}

\begin{abstract}
RESUMEN: Este artículo intenta reunir lo que se ha escrito a propósito de la participación de Rogelio de Tosney en la reconquista ibérica y ampliar lo conocido usando las dos fuentes narrativas que existen: el Chronicon de Adémar de Chabannes y el Chronique de Saint Pierre le Vif. El artículo narra los acontecimientos en que esta implicado este normando en la reconquista ibérica, intentando responder, basándose en estas dos fuentes, preguntas como las razones detrás de su participación, lo qué él alcanzó a conseguir en el condado de Barcelona, y las fechas de los acontecimientos narrados.
\end{abstract}

PALABRAS CLAVE: Crónicas, invasiones, conquista, reconquista, Normandia, Iberia, Barcelona.

ABSTRACT: This article tries to gather what has been written on the subject of Roger of Toeney participation on the Iberian Reconquest and extend it by using the two narrative sources which exist: the Chronicon of Adémar of Chabannes and the Chronique de Saint Pierre le Vif. The article narrates the events of this Norman involvement in the Iberian Reconquest, trying to answer questions, based on these two sources like the reason behind his participation, what did he achieve in the county of Barcelona, and the dates of the events narrated.

KEY WORDS: Chronicles, Invasions, Conquests, Re-conquest, Normandy, Iberia, Barcelona.

Aunque el santuario de Santiago de Compostela se estaba convirtiendo a principios de siglo XI en el lugar preferido de los normandos que viajaban del ducado francés a la península ibérica, el primer normando en participar directamente en las guerras de reconquista, específicamente en el bando cristiano, lo hizo en el condado de Barcelona. ${ }^{1}$ El fue Rogelio de Tosny, un noble normando de gran importancia en el ducado, que a diferencia de muchos de sus compatriotas que vinieron en las décadas siguientes, pertenecía a la alta nobleza normanda. $L a$ participación de este normando no se puede explicar como se suele hacer con los participantes normandos en otras campañas militares, en las cuales estos normados

${ }^{1}$ Storrs, C. M., (1994). Jacobean Pilgrims from England to St. James of Compostella. Santiago de Compostela: Xunta de Galicia. pp. 35-37; 
eran segundos o terceros hijos que no tenían herencia garantizada y por esto buscaban fortuna fuera del ducado. ${ }^{2}$ Evidentemente el caso de Rogelio es algo especial en ese sentido, al menos hasta la participación de su compatriota Rotrou de Perche a principios del siglo XII. Lógicamente por ser el primero en una larga lista de normandos que intervinieron en los conflictos ibéricos, este artículo intentará crear una reseña histórica de los eventos y su intervención, basándose principalmente en las dos crónicas que narran su participación: La Crónica de Adémar Chabannes y la Crónica de San Pablo el Vif de Sens. ${ }^{3}$ Desde luego, también se basará en las crónicas de Olegario Vitalis ${ }^{4}$ y Guillermo de Jumièges ${ }^{5}$ que en el siglo XII reflejaron la imagen que este normando, con su participación temprana en Iberia, pudo reflejar en los ideales y deseos de la nobleza de aquel tiempo.

Primero hay que sopesar las dos fuentes narrativas que se usarán en este artículo teniendo en cuenta su origen y la época en que fueron escritas. La más importante de las dos, para el propósito de este tema, es sin duda la crónica de Adémar de Chabannes por su proximidad no solo geográfica sino también en cuanto a su cercanía temporal a los eventos narrados en su crónica, que fue terminada alrededor del año 1034. ${ }^{6}$ Adémar era un monje escribano de Aquitania, en donde estuvo bastante envuelto en las disputas eclesiásticas de su época. Aunque se ha descubierto que tergiversó la historia para adelantar los intereses de su comunidad, en la mayoría de ocasiones fue un gran cronista que dedicó mucha parte de su

${ }^{2}$ La siguiente participación de Normandos se dio en Barbastro in 1064. See: ArCHER, T.A. (1903). «Giffard of Barbastre», English Historical Review. pp. 303-305; BENNETT, M. (2003). «The Normans in the Mediterranean», A Companion to the Anglo-Norman World. Harper-Bill C. \& van Houts, E. (eds).Woodbridge: Boydell. pp. 87-102; BoISSONNADE, P. (1970). «Les premères croisades français en Espagne», Bolletin Hispanique. pp. 5-28; BoIsSONNADE, P. (1932). «Cluny, la papauté et la premire grande croisade internationale contre les sarrasins d'Espagne-Barbastro», Reveu des questions historiques. pp. 257-301; FERREIRO, A. (1983). «The siege of Barbastro 1064-65: a reassessment», Journal of Medieval History. pp. 129-144; GOÑI GAZTAMBIDE, J. (1958). Historia de la bula de cruzada en España. Vitoria: Editorial del Seminario. pp. 47-49.

${ }^{3}$ CLARIUS DE SENS. (1979). Chronique de Saint-Pierre-le-Vif de Sens. R.H. Boutier \& M. GILleS. (eds). Paris: CNRH. p. 112; Ademari CABAnENnsis. (1999). Chronicon, BOURGain, P., LANDEs, R. y PON, G. (eds). Turnhout: Brepols. p.174.

${ }^{4}$ Orderic Vitalis. (1969). Ecclesiastical History II. Chibnall, M. (ed). Oxford: Clarendon. pp.10-11.

${ }^{5}$ William Of Jumiéges. Gesta Normannorum Ducum. Van Houts, E, (ed). Oxford: Clarendon. pp. 94-97.

${ }^{6}$ LANDES, R. (1995). Relics, Apocalypse, and the Deceits of History: Ademar of Chabannes, 9891034. Cambridge, Mass: Harvard. pp. 125-137. 
investigación a poner los eventos en una cronología lógica basándose en su gran habilidad para encontrar y fechar documentos originales. ${ }^{7}$ Con relación a los eventos de la participación de Rogelio de Tosny en el condado de Barcelona, la crónica de Adémar puede ser una fuente muy confiable ya que el normando, por no ser de Aquitania, no generaría ningún interés por parte de Adémar en tergiversar los eventos narrados, aparte de la típica exageración de las narraciones sobre las guerras con los musulmanes. ${ }^{8}$ Además es probable que Rogelio haya pasado por el monasterio donde Adémar vivía en su viaje de ida o de venida del condado de Barcelona, y por lo tanto sus fuentes pudieron ser de primer orden. Del otro lado la Crónica de San Pedro el Vif de Claricus de Sens fue escrita a principios del siglo XII, extendió la historia del normando y poniendo algunos detalles que no estaban narrados por Adémar, que no siempre son de confiar. ${ }^{9}$ De todas formas, aunque la narración es muy similar a la de Adémar, no es exactamente igual, con detalles que dan la impresión de que, aunque el relato de Adémar pudo ser una fuente de esta crónica mas tardía, es posible que haya tenido otras fuentes de origen. Por la importancia de ambas fuentes, es imperativo darles una mirada para referirse a ellas con mayor precisión en los párrafos siguientes.

La referencia de la crónica de Adémar de Chabannes:

'Item Nortmanni duce Rotgerio, ad occidendos paganos Hispaniam profecti, inumeros Sarracenorum develerunt, et civitates vel castella ab eis abstulere multa. Primo vero adventu suo Rotgerius, Sarracenis captis, unumquemque eorum per dies singulos, videntibus ceteris, quasi porcum per frustalfrustra dividens in caldariis coctum eis opponebat pro epulis, et in alia domo simulabat se comedere cum suis reliqua medietatis membra. Percursis omnibus hoc modo, novissimum de custodia quasi neglegens permittebat fuge, qui haec monstra Sarracenis nunciaret, ita fabulam Tiestis veram adimplens. Qua de causa timore examinati, vicine Hispanie Sarraceni cum rege suo Museto pacem a comitissa Barzelonensi Ermensende petunt, et annuum tributum persolvere spondent. Erat enim haec vidua, et Rotgerio suam filiam in matrimonium socieverat. Cum quibus pace inita, cum ulteriori Hispania Rotgerius decertare cepit, et die quadam una cum Petro episcopo Tolose et XL solummodo Christianis quingentos Sarracenorum electos in insidiis latentes offendit, cum quibus confligens, fratrem

${ }^{7}$ Ibid.

${ }^{8}$ Bissonnade, P. (1970). «Les Primers crosiades françaises en Espagne», en Boletin Historique, pp. 5-7.

${ }^{9}$ Musset, L. (1977). «Aux origines d'une classe dirigeante: les Tosny, grands barons normands du $\mathrm{X}^{\mathrm{e}}$ au XIII ${ }^{\mathrm{e}}$ siècle», en Francia V. pp. 51-53; Clarius De Sens. (1979). Chronique de SaintPierre-le-Vif de Sens. R.H. BOUTIER \& M. GILles (eds) Paris: CNRH. p. vii-xi. 
suum manzerem amisit, tercioque acies adversarias irrumpens plus centum bostium extinxit, et cum suis propria revisit, nec ausi sunt Hismabelite persequi fugientem. ${ }^{10}$

\section{La Cronica de San Pedro de Vif:}

'In illis diebus, Rotgerius, filius Rodulfi comitis, de Normannia perrexit eum exercitu in Hispaniam, vastans ipsam terram capiensque ibi imitates et castella et accipiens uxorem, sororem Ragmundi Berengerii, Stephaniam, quam post eum duxit uxorem rex Hispanie Garsias. Cepit autem imitates Terraconam et Gerundam et habitavit ibi eum uxore et exercitu suo per XV annos. Post hec, homines pagi illius, insidiantes eill, voluerunt eum occidere in ecelesia Sancti Felicis, tempore Richardi Normannorum ducis. Videns autem Rotgerius quod sibi insidias tendebant, relictis XX. viris et uxore et omnibus que possidebat - totum enim suum exercitum jam pene interfecerant - rediit ad patrem suum in Normanniam, cum duce Richardo faciens concordiam. Dolebat enim Richardus de exercitu quem in Hispaniam duxerat quoniam omnes ibi pene interfecti erant. Post hec iste Rotgerius contra [fol. 65 vo] quemdam vicinum faciens bellum interfectus est et multi alii cum eo. ${ }^{11}$

${ }^{10}$ Los normandos, de todas formas, bajo su líder Rogelio hicieron matar a los paganos en España, destruyeron un gran número de sarracenos y tomaron muchas ciudades y fortificaciones de ellos. Cuando Rogelio llegó primero, capturó a los sarracenos, cortó uno de ellos en dos partes, y cada día, y con el resto observando, hirvió una parte en agua como cerdo y luego les dio esta parte como comida, y con la otra parte pretendió ir a comerla a casa con sus hombres. Habiendo tratado a todos de esta forma, él permitió que los mas recientes cautivos escapasen su custodia, haciendo que pareciese negligencia, así que su monstruosa reputación fuese revelada a los sarracenos. Estas noticias llenaron de tal temor a los Sarracenos de la vecina España, que junto con su rey Masatus, buscaron paz con Ermesinda, condesa de Barcelona, y prometieron pagar un tributo anual. Por cierto, Ermesida era una viuda que había casado a su hija con Rogelio. Cuando la paz fue establecida entre ellos, Rogelio resolvió hacer la guerra a un área más de España y un día, con Pedro Obispo de Tolosa y con sólo cuarenta cristianos, fue emboscado por un grupo selecto de quinientos sarracenos que lo esperaban. Luchando con ellos, él perdió a su hermano ilegítimo y entró en la batalla tres veces, mató a cien de sus enemigos y retornó con sus hombres. Los sarracenos no se atrevieron a perseguirle en su fuga.

VAn HouTs, E. (2000). The Normans in European History. Manchester: Manchester University Press. p. 270; Ademari Cabanennsis. (1999). Chronicon, Bourgain, P., Landes, R. y Pon, G. (eds). Turnhout: Brepols. p.174.

${ }^{11}$ En este tiempo, Rogelio hijo de Raúl, vino de Normandía y ganó a España con su hueste, devastando este país y ocupando ciudades y castillos; tomó como esposa a Estefanía la hermana de Ramón Berenguer que se caso después con el Rey García de España (García Sánchez III de Navarra). Tomó las ciudades de Tarragona y Girona, y estuvo allí con su ejército durante quince años. Después de esto, los habitantes de este país, prepararon una emboscada para el y le querían matar en la iglesia de San Félix, durante el tiempo en que Ricardo era duque de Normandía. Pero Rogelio, sabiendo de la emboscada, estaba preparado y dio a perder veinte hombres, su esposa y todo lo que tenía -pues su ejército ya había sido aniquilado casi en su totalidad- y volvió a su padre en Normandía e hizo las paces con el Duque Ricardo (Ricardo II de Normandía). Ricardo, ciertamente, sintió mucho la pérdida de el ejército que el (Rogelio) había llevado a España, donde casi todos habían sido muertos. Clarius De SEns. (1979). Chronique de Saint-Pierre-le-Vif de Sens. R.H. Boutier \& M. Gilles. (eds). París: CNRH. p. 112. 
Rogelio de Tosny como se ha dicho, pertenecía a una de las grandes familias hacendadas de Normandía y que había estado bastante cerca a la casa ducal en las últimas décadas del siglo $\mathrm{X} ;{ }^{12}$ por lo tanto, sus razones para participar en la reconquista no pudieron ser las típicas de la baja y mediana nobleza, de hijos segundos y terceros que no tenía derecho a patrimonio de herencia en casa y que por lo tanto se lanzaban a la aventura en busca de riqueza en otros lugares. Rogelio, queda claro en la Crónica de San Pedro de Vif, que fue desterrado por alguna disputa con el Duque Ricardo; quizás como Musset sugirió, al mismo tiempo que su padre Raúl. ${ }^{13}$ La razón por la cual fue desterrado no es clara, pero se deduce que era bastante poderoso para haber partido en esta aventura con ejército quizá propio y parcialmente hecho de miembros de la baja nobleza. ${ }^{14}$ Ahora, la fecha de su partida no es clara, Musset dice que fue en 1018, pero la última edición de la Crónica de Adémar de Chabannes de Bourgain calcula que la fecha que Adémar definió es el año $1023 .{ }^{15} \mathrm{Y}$ mas problemático aún, el cronista normando Guillermo de Jumièges dice: 'Hic uero (Roger), dum Rodbertus dux peregre perrexerat, in Hispaniam, ubi per eum multe probitates super paganos facte sunt, profectus fuerant. ${ }^{16}$ Y el duque Roberto III partió en su peregrinación hacia tierra santa en el año 1035; mientras que el más conocido cronista normando, Orderico Vitalis, dice que en este año Rogelio fundó la abadía de Conches $;{ }^{17}$ algo que está confirmado en la documentación diplomática de Normandía. $^{18}$ Por estas conflictivas declaraciones de los cronistas, es difícil precisar las fechas de la participación de Rogelio de Tosny en Barcelona, pero por lo menos es claro que la afirmación de la Crónica de los Duques de Normandía de Guillermo de Jumièges no es cierta, porque Adémar murió en 1034 y sería lógicamente imposible para él,

12 Musset, L. (1977). «Aux origines d'une classe dirigeante: les Tosny, grands barons normands du $\mathrm{X}^{\mathrm{e}}$ au XIII ${ }^{\mathrm{e}}$ siècle», en Francia V. pp. 49-51; PotTs, C. (2003). «Normandy 911-1144», en $A$ Companion to the Anglo-Norman World, HARPER-BILl, C. \& VAN HouTs, E. (eds). Woodbridge: Boydell. pp. 19-42.

${ }^{13}$ MusSET. «Les Tosny, grands barons normands du $\mathrm{X}^{\mathrm{e}}$ au XIII ${ }^{\mathrm{e}}$ siècle», en Francia V. pp. 51-52.

${ }^{14}$ Chibnall, M. (1982). «Military Service in Normandy Before 1066», Anglo-Norman Studies $V$. pp. 71-77.

${ }^{15}$ MusSET. «Les Tosny, grands barons normands du $\mathrm{X}^{\mathrm{e}}$ au XIII ${ }^{\mathrm{e}}$ siècle», en Francia V. p. 52.

${ }^{16}$ William Of JumiéGes. Gesta Normannorum Ducum. Van Houts, E, (ed). Oxford: Clarendon. pp. 94-97.

${ }^{17}$ Orderic Vitalis. (1969). Ecclesiastical History II. Chibnall, M. (ed). Oxford: Clarendon. pp. $10-11$

${ }^{18}$ MusSET. «Les Tosny, grands barons normands du $\mathrm{X}^{\mathrm{e}}$ au XIII" siècle», en Francia 5. p. 52. nota 31. 
haber narrado los episodios de Rogelio en Iberia. Desde luego, es posible que el Rogelio al cual se refiera Adémar en su crónica no fuese Rogelio de Tosny, sino otro normando, pero esto sería bastante improbable porque si fuese un aventurero de poca importancia, no hubiese sido de interés para el monje aquitano mencionar y para las otras crónicas referirse; además de que queda bastante claro que Rogelio era conocido en Normandía por haber ido a Hispania. Volviendo a la última fecha calculada sobre la participación de Rogelio según la más moderna edición de la Crónica de Adémar que dice ser el año 1023, parece algo atractivo, por que fue en este año en que el joven Berenguer Ramón I asumió el poder en el condado de Barcelona al cumplir la mayoría de edad. Pero es muy probable que su conocida y hábil madre Ermesinda aún mantuviese control del condado y por esto Adémar no se refirió al joven conde en su crónica. ${ }^{19}$ Del otro lado, la más tardía Crónica de San Pedro el Vif se refiere directamente al Conde, pero se equivoca en el orden de los nombres llamándolo Ramón Berenguer como su mas conocido hijo; quizás por el limitado conocimiento del escribano sobre la historia Barcelonesa. ${ }^{20}$ De todas formas, la Crónica de San Pedro el Vif no menciona a la Condesa Ermesinda, algo que se pude deber a la parcialidad de los conocimientos del cronista francés del área de Sens.

De acuerdo al historiador francés Dozy, que trabajó a finales del siglo XIX en el tema de la participación de este normando en Iberia, Rogelio comenzó su carrera militar, con una serie de razzias o cabalgadas a los territorios controlados por los musulmanes en los alrededores del condado de Barcelona. El trato que supuestamente el normando les daba a sus prisioneros, tenía como objetivo crear tal terror a sus enemigos, que los viese obligados a dar un tributo en forma de parias a la condesa Barcelonesa, para evitar caer en las manos del cruel normando. ${ }^{21}$ De acuerdo a Adémar, el rey de los sarracenos Masetus, que los historiadores franceses tradicionalmente lo identifican como Modjehid b. Yasuf rey

${ }^{19}$ Urbina Moterell, F. (1958). «Los Condes Catalanes: Prosopografía», en La Reconquista y el proceso de diferenciación política (1035-1217), LADERO QueSADA, M. L. (ed). Madrid: Espasa Calpe. pp. 341-342; Aurell I CARDONA, M. (1993). «Les avatars de la viduité princière: Ermessende (ca. 975-1058), comtesse de Barcelone», en Veuves et veuvage dans le haut moyen âge, PARISSE, M. (ed). Paris: Picard. pp. 201-215.

${ }^{20}$ Clarius De Sens. (1979). Chronique de Saint-Pierre-le-Vif de Sens. R.H. Boutier \& M. GILLES. (eds). Paris: CNRH. p. 112.

21 Ademari Cabanennsis. (1999). Chronicon, Bourgain, P., Landes, R. y Pon, G. (eds). Turnhout: Brepols. pp. 173-174; DOZY, R.(1881). Recherches sur l'historie et la littérature de l'Espagne pendant le Moyen Age II. Paris: Maissoneve \& Co. p. 334; MusseT. «Les Tosny, grands barons normands du $\mathrm{X}^{\mathrm{e}}$ au XIII ${ }^{\mathrm{e}}$ siècle», en Francia V. pp. 49-51 
de Denia, es probable, que este rey de Taifa le diese tributos o parias a la condesa regente o a su hijo, ya que esto era común en la época; pero que haya sido la actuación de Regelio de Tosny y su ejército los que hayan precipitado tal acontecimiento, no está claro en la documentación barcelonesa de la época que en ningún documento encontrado hasta el momento parece referirse a este normando, algo que hace bastante problemático el estudio de su estancia en lares de Barcelona. $^{22}$

De otro lado, las otras conquistas mencionada por la Crónica de San Pedro el Vif como son las de Tarragona y Girona, parecen más fabricaciones del escribano o interpolaciones que realidad, ya que Girona estaba bajo dominio Cristiano y si la hubiese conquistado, este episodio hubiese quedado registrado en los anales de la historia de la región, cosa que no está y que Adémar no menciona. El caso de Tarragona también es algo problemático, aunque si es posible que haya hecho razzias en el área, ya que se sabe que Tarragona estuvo prácticamente abandonada hasta su repoblación por el aventurero, también normando, Roberto Burdet, en la primera mitad del siglo XII; aunque a finales del siglo XI ya había habido intentos de repoblación de la grande y antigua capital romana. ${ }^{23}$ Por lo tanto es probable que Rogelio haya tratado de reconquistar la ciudad abandonada, pero es imposible saber si en realidad se estableció allí como afirma la Crónica de San Pedro el Vif.

Otra área a la cual se refieren las dos crónicas mencionadas sobre la carrera de este normando en Barcelona, es su supuesto matrimonio con la hija de Ermesinda o hermana del conde, después de sus hazañas, para cimentar su alianza con la casa condal. Musset ha sido muy escéptico con la existencia de este matrimonio habiendo asegurado: "Au XII siècle l'annaliste Clarius de Sens prétend savoir que la femme de Roger se nommait Stéphanie et qu'elle épousa plus tard «le Roi de Espagne Garsias», sans doute Garcia III de Navarre.",24 Musset creyó que en realidad Clarius erróneamente escogió a Estefanía la hija de Ermesinda, que como él dijo luego, se casó García Sánchez rey de Navarra. Es posible que en realidad esto haya sido como el dijo, una creación del monje senense, pero el mismo

${ }^{22}$ MuSSET. «Les Tosny, grands barons normands du $\mathrm{X}^{\mathrm{e}}$ au XIII ${ }^{\mathrm{e}}$ siècle», en Francia 5. p. 52.

23 Benito Ruano, E. (1994). «El principado de Tarragona», Misel-lània Ramon d'Abadal; Estudis Universitaris Catalans. pp. 107-119; MCCRANK, L. J. (1981). «Norman Crusaders in the Catalan Reconquest: Robert Burdet and the Principality of Tarragona», Journal of Medieval History. pp. 67-82; MiRET Y SANS, J., (1933). «La familia de Robert Burdet, el restaurador de Tarragona», Segundo congreso de historia de la corona de Aragón: actas y memorias I. pp. 53-74; NelsON, L. (1970). «Rotrou of Perche and the Aragonses Recoquest» Traditio, pp. 113-133.

${ }^{24}$ MusseT, «Les Tosny, grands barons normands du X ${ }^{\mathrm{e}}$ au XIII ${ }^{\mathrm{e}}$ siècle», en Francia V. p. 52. 
Adémar afirmó que Rogelio contrajo matrimonio con una hija de la condesa de Barcelona, cosa que Musset acepta, pero pone en entredicho que la esposa con que Rogelio aparece en los diplomas normandos llamada Godehildis, se trate de una barcelonesa; ya que de haber existido tal unión, los cronistas normandos como Orderico Vitalis, hubiesen escrito sobre este matrimonio. Pero hay algo que queda claro de la Crónica de San Pedro el Vif y es que Rogelio abandonó a su esposa cuando regresó a Normandía, algo a lo cual Musset no dio atención. Si esto fue así, es posible que verdaderamente Rogelio pudo estar comprometido o casado con Estefanía o con otra hija de Ermesinda durante su estadía en Barcelona. ${ }^{25}$ Por no haber traído a su esposa de regreso a Normandía, no hubiese sido del conocimiento o del interés de los cronistas normandos, que a su juicio, quizás la separación de Rogelio de su esposa peninsular lo hubiese dejado con no muy buenas relaciones con su ex-suegra y con su ex-cuñado, y por lo tanto la alianza matrimonial hubiese perdido su sentido original.

El tiempo de la estadía de Rogelio en el condado de Barcelona fue calculado por Clarus de Sens de haber sido 15 años, mientras Adémar no precisó cuánto tiempo estuvo el normando. ${ }^{26}$ Es difícil conciliar la declaración de Clarus de Sens con las posibilidades sobre la estadía de Rogelio en el condado de Barcelona, pues si los eventos sucedidos acontecieron durante el gobierno del Duque Ricardo II (muerto en 1026) o incluso durante el gobierno de Ricardo III (muerto en 1028), él tendría que haber partido a Hispania en 1013 y ya se ha calculado que él partió después de 1018 en su destierro temporal..$^{27}$ Por lo tanto, es probable que la exhortación de Clarius con respecto al tiempo que estuvo este normando en el condado mediterráneo fue una exageración. Si se aproxima que Rogelio regresó a Normandía antes de la muerte de uno de estos dos duques, es probable que estuviese menos de diez años, ya que como se ha dicho, no existe ninguna documentación conocida sobre la carrera de este normando en Barcelona.

Rogelio de Tosny terminó su estadía en el condado de Barcelona abruptamente como se ha dicho en ambas crónicas, después de una derrota militar infringida por los habitantes de la región. En esta derrota, que las crónicas mencionan con alguna similitud narrativa, sus tropas son diezmadas y de acuerdo con Adémar, Rogelio

25 Aurell I CARDOnA, M. (1993). «Les avatars de la viduité princière: Ermessende (ca. 9751058), comtesse de Barcelone», en Veuves et veuvage dans le haut moyen âge, PARISSE, M. (ed). Paris: Picard. pp. 214-215.

${ }^{26}$ Clarius De Sens. (1979). Chronique de Saint-Pierre-le-Vif de Sens. Boutier, R.H. \& Gilles, M. (eds). Paris: CNRH. p. 112.

${ }^{27}$ BAtes, D. (1982). Normandy Before 1066. London: Longman. pp. 99-106. 
perdió a su hermano ilegítimo. ${ }^{28}$ Queda claro que junto a Rogelio había un número de normandos de mas baja categoría que formaban su ejército y no es de sorprenderse que un hermano ilegítimo estuviese con él en el exilio, quizás buscando riqueza y estado ya que en Normandía sus posibilidades eran más limitadas. Aunque su hermano falleció en esta emboscada quizás hubo otros combatientes mas afortunados como el caballero hijo de Vexin Nicolas Fils Baudry, al cual se refiere Musset en su articulo, de haber participado en una expedición a Hispania, que probablemente fue la de Rogelio de Tosny. ${ }^{29}$

Volviendo entonces a la derrota final, es claro desde el punto de vista de Adémar, que aunque el normando y su ejercito fueron derrotados por el ejército de los sarracenos, la Crónica de San Pedreo el Vif no aclara si los que atacaron al normando en esta batalla final fueron sarracenos o cristianos, simplemente refiriéndose a ellos como la gente de este país. ${ }^{30} \mathrm{Si}$ fueron los sarracenos, sería de esperarse, pues queda claro que inclusive con al acuerdo de tributo que los musulmanes prometieron, Rogelio continuó una política agresiva hacia ellos que seguro terminaría antagonizando hasta a los cristianos barceloneses, ya que la guerra continuada no era provechosa para ninguno de los dos bandos ibéricos. Es posible que la nobleza local tuviese recelos de un extranjero que estaba tan cerca de la familia condal y que, por lo que narran las crónicas, parecía tener una política de continuas razzias de los territorios musulmanes. Lógicamente la envidia y la falta de estima que es muy seguro que sentían la nobleza ibérica en relación con los participantes extranjeros en la península durante todo el proceso de reconquista, se ha visto descrita en otros episodios de la historia de estos conflictos, como en la primera supuesta participación de Rotrou de Peche en Aragón a principios del siglo XII que Orderico Vitalis describió en su crónica. ${ }^{31}$ El antagonismo era suficientemente real para haber causado la expulsión de Rogelio de Tosny del condado, pero lo mas probable es que en realidad su ejército fue diezmado por los musulmanes que Adémar mencionó en su crónica; Rogelio decidió que ya había

28 'fratrem suum manzerem amisit.' ADEMARI CABANENnSIS. (1999). Chronicon, Bourgain, P., LANDES, R. y PON, G. (eds). Turnhout: Brepols. p.174.

${ }^{29}$ Musset. (1977). «Les Tosny, grands barons normands du X ${ }^{\mathrm{e}}$ au XIII ${ }^{\mathrm{e}}$ siècle», en Francia V. pp. 52-53. Fauroux, M. (ed). (1961). Recueil des Actes des ducs de Normandie 911-1066. Caen: la Société des Antiquaires de Normandie. Núm. 102. p. 259.

${ }^{30}$ Clarius De Sens. (1979). Chronique de Saint-Pierre-le-Vif de Sens. Boutier, R.H. \& Gilles, M. (eds). Paris: Cnrh. p. 112; Ademari CABAnennsis. (1999). Chronicon, Bourgain, P., Landes, R. y PON, G. (eds). Turnhout: Brepols. p.174.

${ }^{31}$ Ordericus Vitalis. (1972). Ecclesiastical History VI. Chibnall, M (ed). Oxford: Clarendon. pp. 394-397; 
conseguido suficiente riqueza y volvió a Normandía donde se reconcilió con el duque, quizás ofreciéndole algo de la riqueza adquirida durante su estadía en Iberia.

La participación de Rogelio no fue la primera participación de normandos en la península ibérica pues desde el siglo IX es seguro que grupos de vikingos habían ya atacado y saqueado las costas de la península. ${ }^{32}$ Inclusive, relativamente contemporáneo a los eventos de la participación de Rogelio de Tosny en Barcelona, un grupo de vikingos ataco las costas probablemente de Galicia e inclusive a Lisboa. ${ }^{33}$

Pero ciertamente Rogelio de Tosny fue el primer normando cristiano en participar en las campañas de la reconquista ibérica, específicamente del lado cristiano. Auque la narrativa histórica de este personaje es difícil de descifrar y no puede haber total certeza con respecto a sus hazañas en el condado de Barcelona, su participación sirvió muy probablemente de ejemplo a otros normandos, incluyendo a su hijo Ralph, para participar en las guerras de reconquista. ${ }^{34}$ Lógicamente su participación es improbable que tuviese una motivación de cruzada, ya que la idea aún no se había gestado en el pensamiento de la época, pero la idea de guerra santa no es improbable que pudo haberle servido a Rogelio y especialmente a sus sucesores, para engrandecer la imagen de la familia en su historia local. Ciertamente Rogelio vino a ser conocido en la historiografía normanda como Rogelio el Hispánico, lógicamente por sus aventuras en Iberia que muy seguramente le sirvieron para equipararse con las populares historias de Roldán que ya se hacían famosas en Normandía en el siglo XI. Es por esto que es probable que aunque el impacto de las aventuras de Rogelio en la Península Ibérica no causaron una reacción inmediata de participantes normandos en las campañas de la reconquista, la intervención de los normandos comenzó gradualmente a intensificarse hasta tener las grandes participaciones de Normandos y AngloNormandos del siglo XII. ${ }^{35}$

${ }^{32}$ Ferriro Alemparte, J. (1999). Arribadas de normados y cruzados a las costas de la península ibérica. Madrid: Sociedad Española de Estudios Medievales. pp. 19-52.

${ }^{33}$ Dudo De St Quentin. (1998). History of the Normans. Christiansen, E. (ed). Woodbridge: Boydell. p. 162

${ }^{34}$ Ordericus Vitalis. (1967). Ecclesiastical History III. ChiBnAll, M. (ed). Oxford: Clarendon. p. xix.

${ }^{35}$ Deforneaux, M. (1949). Les Français en Espagne. Paris: Presses Universitaries. 125-257. 